\title{
THE HAUSDORFF DIMENSION OF THE BOUNDARY OF THE LÉVY DRAGON
}

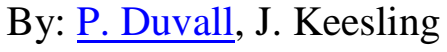

\begin{abstract}
:
A theoretical approach to computing the Hausdorff dimension of the topological boundary of attractors of iterated function systems is developed. The curve known as the Lévy Dragon is then studied in detail and the Hausdorff dimension of its boundary is computed using the theory developed. The actual computation is a complicated procedure. It involves a great deal of combinatorial topology as well as determining the structure and certain eigenvalues of a $752 \times$ 752 matrix. Perron-Frobenius theory plays an important role in analyzing this matrix.

\section{Introduction}

In [L1], P. Lévy studied the complex curve which has come to be known as the Lévy Dragon. He showed that the Dragon is self-similar, and argued that the plane can be tiled by copies of it. We give a self-contained account of the Dragon that determines the properties of this set that we need in our computations. However, the paper by Lévy is a tour de force on this subject. It is amazing that he was able to determine so many properties of this and other curves in [L1] without the use of modern computers. The reader is urged to see [L2] for an excellent annotated translation of [L1].
\end{abstract}

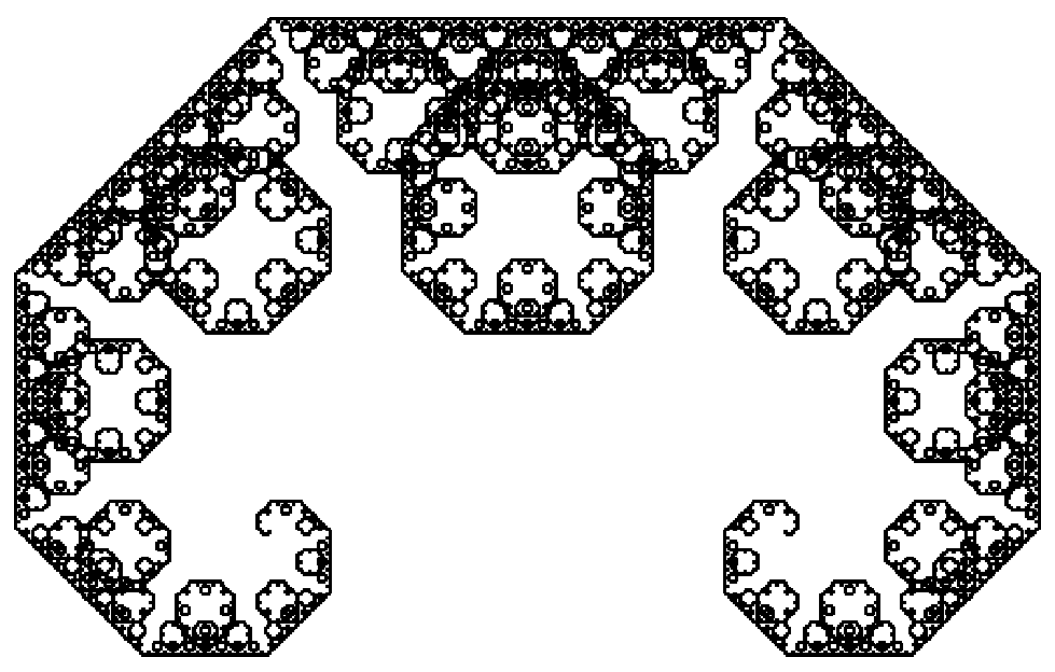

1991 Mathematics Subject Classification. Primary 28A20, 54E40, 57Nxx.

Key words and phrases: Hausdorff dimension, iterated function systems, attractors, fractal geometry.

This research was done while the first author was a Visiting Professor at the University of Florida. He gratefully acknowledges UFL's hospitality, as well as research support from the University of North Carolina, Greensboro.

Typeset by AMS-TEX 


\section{Figure 1. The Lévy Dragon}

Although Lévy found so many properties of the Levy Dragon, he did not compute the Hausdorff dimension of its boundary. In [L2, page 236] G. Edgar asks whether the dimension of the boundary of the Dragon is greater than one. In this paper we answer that question in the affirmative and in fact determine a precise value for this dimension. In the process of doing this we develop a general theory for computing the Hausdorff dimension of the boundary of self-similar fractal tiles. This depends heavily on the paper by K. Falconer [F1] who gave an (abstract) method for determining the Hausdorff dimension of sub-self-similar sets. Our contribution is to show that in the case of boundaries, it is possible to derive a method which can be carried to a computation. We then make a detailed study of the Lévy Dragon and show how to apply our theory to compute the dimension of its boundary. In subsequent work the authors together with A. Vince have found a general method for finding the dimension of the boundary of self-similar digit tiles [DKV]. The size of the matrices involved in computing the dimensions of the boundaries of these tiles is typically less than $10 \times 10$. Unfortunately the Lévy Dragon is not a self-similar digit tile and so these methods do not apply directly to this example. Fortunately, motivated by some ideas in this paper R. Strichartz and Y. Wang [SW] found a method of computing the dimension of the boundary of the Lévy Dragon which has considerably reduced the computations required in this case as well. In their method one only needs to determine a certain eigenvalue of an $11 \times 11$ matrix. Strichartz and Wang made use of the graph directed constructions of D. Mauldin and S. Williams [MW] rather than the sub-self-similar sets of Falconer [F1] used in this paper and in [DKV].

First, we need to establish some terminology and notation. We assume that the reader is familiar with the basic theory of self-similar fractals. The books by Edgar [E1] and Falconer [F2] are excellent references. The book [E2] contains a number of papers which give a nice historical perspective on the subject.

If $X$ is a complete metric space and $F=\left\{f_{1}, f_{2}, \cdots, f_{n}\right\}$ is a collection of contraction mappings of $X$ to itself, then $F$ is said to be a (hyperbolic) Iterated Function System or IFS. It is well known $[\mathrm{H}]$ that for such an $F$ there is a unique compact set $K$ such that $K=\bigcup_{i=1}^{n} f_{i}(K) . K$ is called the attractor or invariant set for $F$. In fact, if $\mathcal{C}(X)$ denotes the collection of non-empty compact subsets of $X$ with the Hausdorff metric, then the map $F: \mathcal{C}(X) \rightarrow \mathcal{C}(X)$ given by $F(A)=\bigcup_{i=1}^{n} f_{i}(A)$ is a contraction mapping. Thus $K$ is the unique fixed point of $F$ guaranteed by Banach's contraction mapping principle, and $K=\lim _{k \rightarrow \infty} F^{k}(A)$ for any $A \in \mathcal{C}(X)$. In this paper, we will only be concerned with the case in which the $f_{i}$ are contracting similitudes, maps satisfying $d\left(f_{i}(x), f_{i}(y)\right)=c_{i} d(x, y)$, where $d$ is the metric on $X$ and $0<c_{i}<1$.

Our main interest in this paper is the Lévy Dragon. It is the attractor for the IFS $F=\left\{f_{1}, f_{2}\right\}$, where $f_{1}$ and $f_{2}$ are similitudes of Euclidean space $\mathbf{R}^{2}$ given by

$$
\begin{aligned}
& f_{1}(x, y)=\left(\frac{x-y}{2}, \frac{x+y}{2}\right) \\
& f_{2}(x, y)=\left(\frac{x+y+1}{2}, \frac{y-x+1}{2}\right) .
\end{aligned}
$$


However, much of the theory developed in the early sections holds in a much broader setting. So, it is appropriate to have fairly general notational conventions. The Hausdorff dimension of a set $E$ is denoted by $\operatorname{dim}_{H} E$, and the lower and upper box-counting dimensions of $E$ are denoted by $\underline{\operatorname{dim}}_{B} E$ and $\overline{\operatorname{dim}}_{B} E$. Definitions of of these dimension functions can be found in $[\mathrm{F} 1],[\mathrm{F} 2]$ and $[\mathrm{E}]$.

Let $\Omega$ denote the collection of sequences $I=\left\{i_{1}, i_{2}, \cdots\right\}$ with $i_{k} \in\{1,2, \cdots, n\}$. $\Omega_{k}$ will denote the set of sequences of length $k$ with entries from $\{1,2, \cdots, n\}$. $\Omega$ has the natural metric given by

$$
d(I, J)= \begin{cases}1, & \text { if } i_{1} \neq j_{1} \\ c_{i_{1}} c_{i_{2}} \cdots c_{i_{m}}, & \text { if } i_{k}=j_{k} \text { for } k \leq m \text { and } i_{m+1} \neq j_{m+1} .\end{cases}
$$

For $I \in \Omega$, we let $I_{k}$ denote the finite sequence $\left\{i_{1}, i_{2}, \cdots, i_{k}\right\}$, and will often use the shorthand $f_{I_{k}}$ for the composition $f_{i_{1}} f_{i_{2}} \cdots f_{i_{k}}$ and $c_{I_{k}}$ for the product $c_{i_{1}} c_{i_{2}} \cdots c_{i_{k}}$. We will also need the maps $\sigma: \Omega \rightarrow \Omega$ and $g: \Omega \rightarrow K$ given by $\sigma\left(i_{1}, i_{2}, \cdots\right)=\left\{i_{2}, i_{3}, \cdots\right\}$ and $g(I)=\lim _{k \rightarrow \infty} f_{i_{1}} f_{i_{2}} \cdots f_{i_{k}}(x)$. It is not difficult to see that $g(I)$ is independent of the choice of $x \in X$. The map $\sigma$ is the well-known shift map on the sequence space.

\section{$\S 2$. Sub-Self-Similar Sets}

In [F1], Falconer introduced the notion of sub-self-similar (s.s.s.) sets, and gave an (abstract) way to determine the dimension of such sets. In this section, we give a brief summary, in our notation, of the notions from [F1] that we will need for our calculations. Given a set $F=\left\{f_{1}, f_{2}, \cdots, f_{n}\right\}$ of contracting similitudes on $\mathbf{R}^{l}$, the closed set $E$ is said to be sub-self-similar for $F$ if $E \subset \bigcup_{i=1}^{n} f_{i}(E)$. It follows that $E \subset K$, where $K$ is the attractor for $F$. Note in particular that the topological boundary $\partial K$ of $K$ is s.s.s., since the interior int $K$ of $K$ is mapped into itself by each of the open mappings $f_{i}$. The first crucial observation about s.s.s. sets is

2.1 Proposition. Let $E$ be a closed set. Then $E$ is s.s.s. for $F$ if and only if $E=g(A)$ for some compact set $A \subset \Omega$ such that $\sigma(A) \subset A$. Furthermore, if $E$ is s.s.s., such a set $A$ is given by $A=\left\{I \in \Omega \mid g\left(\sigma^{k}(I)\right) \in E\right.$ for all $\left.k \geq 0\right\}$.

Proof. This is Proposition 2.1 (and its proof) in [F1].

The set $A$ in Proposition 2.1 is fundamental in the calculation of $\operatorname{dim}_{H} E$. For $k>0$, let $A_{k}$ be the set of finite sequences obtained by truncating elements of $A$ after $k$ terms. For $s \geq 0$ define

$$
\tau(s)=\lim _{k \rightarrow \infty}\left(\sum_{I_{k} \in A_{k}} c_{I_{k}}^{s}\right)^{1 / k}
$$

Recall that $F$ satisfies the open set condition $[\mathrm{H}]$ if there is a bounded nonempty open set $U$ such that

$$
\begin{gathered}
\bigcup_{i=1}^{n} f_{i}(U) \subset U, \text { and } \\
f_{i}(U) \cap f_{j}(U)=\emptyset, \text { if } i \neq j .
\end{gathered}
$$


Our main tool for calculating dimensions is the following theorem of Falconer.

2.2 Theorem. Let $E$ be a closed set which is s.s.s. with respect to the family $\left\{f_{1}, f_{2}, \cdots, f_{n}\right\}$ of contracting similitudes which satisfies the open set condition. Let $s$ be the number satisfying $\tau(s)=1$. Then $s=\operatorname{dim}_{H} E=\underline{\operatorname{dim}}_{B} E=\overline{\operatorname{dim}}_{B} E$.

Proof. This is Theorem 3.5 of [F1]. The packing dimension $\operatorname{dim}_{P}(X)$ is currently an important dimension function as well. Since $\operatorname{dim}_{H}(E) \leq \operatorname{dim}_{P}(E) \leq \operatorname{dim}_{B}(E)$ is always satisfied, we also have that $s=\operatorname{dim}_{P}(E)$ as well.

\section{§3. Boundaries of Attractors}

In this section, we specialize to the case of the boundary of the attractor of an $I F S$ satisfying the open set condition, and show that stronger invariance properties of the boundary give a more tractable version of Theorem 2.2 for computing its Hausdorff dimension. Let $F=\left\{f_{1}, f_{2}, \cdots, f_{n}\right\}$ be an $I F S$ satisfying the open set condition, let $K$ be its attractor, and let $A \subset \Omega$ be as in the previous section. One obvious difficulty in applying Theorem 2.2 is that one has to sum over finite sequences determined by an infinite condition. Let

$$
A^{\prime}=\left\{I \in \Omega \mid f_{I_{n}}(K) \cap \partial K \neq \emptyset \text { for all } n>0\right\} .
$$

We will show that $A^{\prime}=A$ and use that to show that membership in $A_{n}$ can be determined from finite data.

3.1 Proposition. $\sigma\left(A^{\prime}\right) \subset A^{\prime}$.

Proof. Suppose that $I \subset A^{\prime}$ but $\sigma(I) \notin A^{\prime}$. Then for some $n$,

$$
f_{i_{2}} f_{i_{3}} \cdots f_{i_{n}}(K) \subset \operatorname{int} K \text {. }
$$

But since each $f_{i}$ maps the interior of $K$ into itself,

$$
f_{i_{1}} f_{i_{2}} \cdots f_{i_{n}}(K) \subset f_{i_{1}}(\text { int } K) \subset \operatorname{int} K
$$

a contradiction.

3.2 Proposition. $A=A^{\prime}$.

Proof. Suppose that $I \in A^{\prime}$. Let $k$ be given, and use $p \in K$ to compute $g$. For any $m>0$, 3.1 implies that $f_{i_{k}} f_{i_{k+1}} \cdots f_{i_{k+m}}(K) \cap \partial K \neq \emptyset$, so

$$
d\left(f_{i_{k}} f_{i_{k+1}} \cdots f_{i_{k+m}}(p), \partial K\right) \leq c_{i_{k}} c_{i_{k+1}} \cdots c_{i_{k+m}} .
$$

Therefore $\lim _{m \rightarrow \infty} f_{i_{k}} f_{i_{k+1}} \cdots f i_{k+m}(p) \in \partial K$ and $I \in A$ and $A^{\prime} \subset A$.

Suppose that $I \in A$, but $f_{i_{1}} f_{i_{2}} \cdots f_{i_{k}}(K) \cap \partial K=\emptyset$. Then

$$
f_{i_{1}} f_{i_{2}} \cdots f_{i_{k}}(K) \subset \operatorname{int} K
$$

Since

$$
f_{i_{1}} f_{i_{2}} \cdots f_{i_{k}}(K) \supset f_{i_{1}} f_{i_{2}} \cdots f_{i_{k+1}}(K) \supset \cdots \supset f_{i_{1}} f_{i_{2}} \cdots f_{i_{k+m}}(K)
$$

for all $m$, it follows that $g(I) \in \operatorname{int} K$, a contradiction. Thus $A \subset A^{\prime}$. 
3.3 Proposition. $A_{k}=\left\{I \in \Omega_{k} \mid f_{I_{k}}(K) \cap \partial K \neq \emptyset\right\}$.

Proof. Let $I_{k}$ be a sequence in $\Omega_{k}$ such that $f_{I_{k}}(K) \cap \partial K \neq \emptyset$. If $f_{I_{k-1}}(K) \subset$ int $K$, we would have that $f_{I_{k}}(K)=f_{I_{k-1}}\left(f_{i_{k}}(K)\right) \subset f_{I_{k-1}}(K) \subset$ int $K$, a contradiction. Thus $f_{I_{k-1}}(K) \cap K \neq \emptyset$, and, working backwards, we get that $f_{I_{j}}(K) \cap K \neq \emptyset$ for $j<k$. Next, we need to show that $I_{k}$ extends to a sequence in $A$. Since $f_{I_{k}}=\bigcup_{j} f_{I_{k}}\left(f_{j}(K)\right)$, there is some $i_{k+1}$ such that $f_{i_{1}} \cdots f_{i_{k}} f_{i_{k+1}}(K) \cap \partial K \neq \emptyset$. Continuing by induction gives the necessary extension of $I_{k}$.

Proposition 3.3 leads to a strategy for computing $\operatorname{dim}_{H} \partial K$. Suppose for convenience that the $c_{i}$ all have the same value $c$, and suppose that we can determine $\alpha=\lim _{k \rightarrow \infty}\left|A_{k}\right|^{1 / k}$, where $|S|$ denotes the cardinality of the set $S$. Then

$$
\tau(s)=\lim _{k \rightarrow \infty}\left(\sum_{I_{k} \in A_{k}} c_{I_{k}}^{s}\right)^{1 / k}=\lim _{k \rightarrow \infty}\left(\left|A_{k}\right| c^{k s}\right)^{1 / k}=\alpha c^{s} .
$$

Thus $\operatorname{dim}_{H} \partial K$ is the solution to $\alpha c^{s}=1$, or

$$
\operatorname{dim}_{H} \partial K=-\frac{\ln (\alpha)}{\ln (c)}
$$

\section{§4. The LÉvy Dragon}

For the rest of this paper, $K$ will denote the Lévy Dragon, which is the attractor of the IFS $F=\left\{f_{1}, f_{2}\right\}$, where $f_{1}$ and $f_{2}$ are the similitudes of Euclidean space $\mathbf{R}^{2}$ given by

$$
\begin{aligned}
& f_{1}(x, y)=\left(\frac{x-y}{2}, \frac{x+y}{2}\right) \\
& f_{2}(x, y)=\left(\frac{x+y+1}{2}, \frac{y-x+1}{2}\right)
\end{aligned}
$$

Lévy [L1] studied $K$ extensively, and showed, among other things, that $K$ tiles the plane in the sense that the plane can be written as the union of congruent copies of $K$ that meet only in their boundaries. It is first important that we establish that $K$ satisfies the open set condition so that we can use Theorem 2.2. The following Lemma can be found in [B] or $[\mathrm{K}]$.

4.1 Lemma. Suppose that $\left\{f_{1}, \ldots, f_{n}\right\}$ is an IFS on $\mathbf{R}^{l}$ with $K$ the invariant set. Suppose that $\sum_{i=1}^{n} c_{i}^{n}=1$. Then if $K$ has nonempty interior in $\mathbf{R}^{l}$, then $K$ satisfies the open sets condition with the int $K$ being the open set.

Proof. Let $U=\operatorname{int} K$. Then since each $f_{i}$ is an open mapping in $R^{l}$, it must be the case that $f_{i}($ int $K) \subset$ int $K$ for each $1 \leq i \leq l$. By Lemma 1.1 of [K] it must be the case that $\mathcal{H}^{l}\left(f_{i}(K) \cap f_{j}(K)\right)=0$ for all $i \neq j$, where $\mathcal{H}^{\alpha}(A)$ denotes the Hausdorff $\alpha$-measure of 
$A$. Since Lebesgue $l$-dimensional measure is proportional to $\mathcal{H}^{l}$, this implies that $\lambda\left(f_{i}\right.$ (int $K) \cap\left(f_{j}(\right.$ int $\left.K)\right)=0$. Of course, this is only possible if $f_{i}($ int $K) \cap\left(f_{j}(\right.$ int $K)=\emptyset$.

For our study, we will need some more notation. Let $S$ be the unit square with vertices $\{(0,0),(0,1),(1,1),(1,0)\}$ and let $T_{0} \subset S$ be the triangle with vertices $\{(0,0),(1 / 2,1 / 2),(1,0)\}$. We will view $K$ as $\lim _{k \rightarrow \infty} F^{k}\left(T_{0}\right)$.

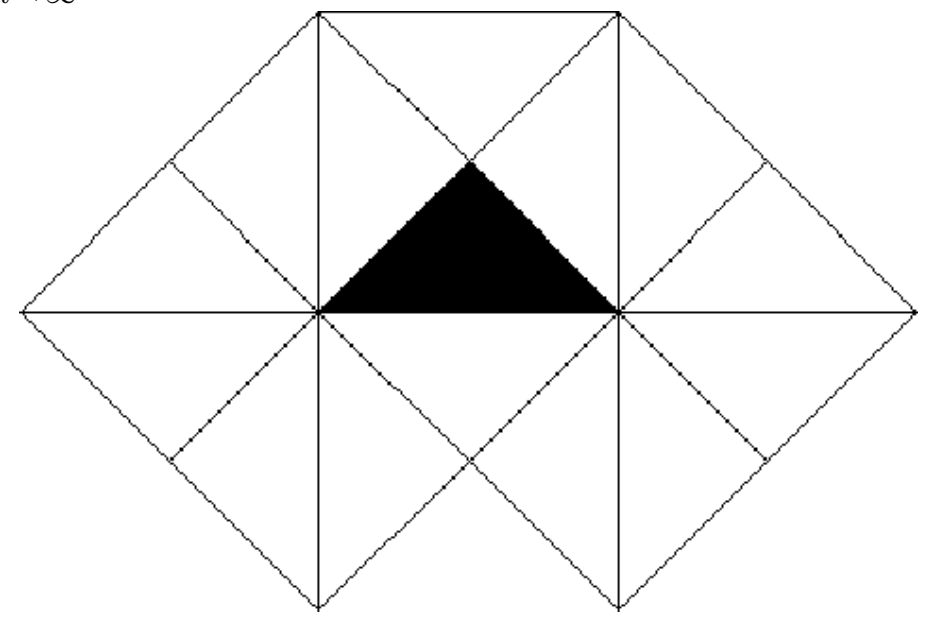

Figure 2. $T_{0}$ And Its Neighborhood

$T_{0}$ and its neighborhood in $\mathcal{T}_{0}$ are shown in Figure 2. $S$ is the union of the four triangles in $S$ with hypotenuse an edge of $S$ and third vertex at $(1 / 2,1 / 2)$. We denote the triangulation of $\mathbf{R}^{2}$ consisting of these triangles and their integral translates by $\mathcal{T}_{0}$. If $T$ is a right triangle in the plane, we refer to the vertex opposite the hypotenuse as the top vertex of $T$, and the other vertices as left and right so that we encounter the vertices in the order left, top, and right as we traverse the boundary in clockwise order. The left(right) edge of $T$ is the edge determined by the left(right) and top vertices of $T$. The triangulation $\mathcal{T}_{0}$ has a subdivision $\mathcal{T}_{1}$ obtained by subdividing each triangle $T$ into the two triangles determined by the left(right) edge of $T$ and the midpoint of the hypotenuse of $T$. Continuing in the obvious way gives a sequence $\mathcal{T}_{0} \succ \mathcal{T}_{1} \succ \cdots \succ \mathcal{T}_{k} \succ \cdots$ of subdivisions of $\mathbf{R}^{2}$ into right isosceles triangles. Each triangle in $\mathcal{T}_{k}$ has diameter $\left(\frac{1}{\sqrt{2}}\right)^{k}$. For each $T \in \mathcal{T}_{k}$, let $T^{L}$ be the triangle in $\mathcal{T}_{k+1}$ whose hypotenuse is the left edge of $T$ and which has a vertex outside of $T$. Define $T^{R}$ similarly. One can easily see that $F\left(T_{0}\right)=T_{0}^{L} \bigcup T_{0}^{R}$. In fact, the following Proposition is immediate.

4.2 Proposition. For each $k>0, F^{k}\left(T_{0}\right)$ is a union of $2^{k}$ triangles in $\mathcal{T}_{k} . F^{k+1}\left(T_{0}\right)$ is obtained from $F^{k}\left(T_{0}\right)$ by replacing each $T$ in $F^{k}\left(T_{0}\right)$ by $T^{L}$ and $T^{R}$.

We would like to use the formula at the end of $\S 3$ to compute the Hausdorff dimension of $\partial K$. The problem is that $K$ and $\partial K$ are very complicated objects, and we can only see finite approximations to them, so it is not clear how to compute $A_{k}$. What we can hope to compute is $B_{k}=\left\{I_{k} \in \Omega_{k} \mid f_{i_{k}}\left(T_{0}\right) \cap \partial F^{k}\left(T_{0}\right) \neq \emptyset\right\}$. We now attack that problem together with the question of relating $A_{k}$ to $B_{k}$.

For $T \in \mathcal{T}_{k}$, let $N(T)$ be the star of $T$, that is, $N(T)=\left\{T^{\prime} \in \mathcal{T}_{k} \mid T \cap T^{\prime} \neq \emptyset\right\}$. Each $N(T)$ consists of 15 triangles. For technical reasons to be seen later, we order these triangles as 
$N(T)[1], N(T)[2], \cdots, N(T)[15]$ as follows: $T=N(T)[1]$; then $N(T)[2]-N(T)[8]$ are the triangles incident with the left vertex of $T$ in clockwise order, so that $N(T)[8]$ has the left edge of $T$ for its right edge; $N(T)[9]$ is incident with the top vertex of $T$ and and has the left edge of $N(T)[8]$ for its right edge; $N(T)[10]$ has the right edge of $T$ for its left edge; and $N(T)[11]-N(T)[15]$ are the remaining triangles incident with the right vertex of $T$ ordered clockwise. This ordering is pictured in Figure 3. The neighborhood type $\rho(T)$ is the 15-tuple $\rho(T)=\left\{\rho_{1}, \rho_{2}, \cdots, \rho_{15}\right\}$, where

$$
\rho_{i}= \begin{cases}1, & \text { if } N(T)[i] \in F^{k}\left(T_{0}\right) \\ 0, & \text { otherwise }\end{cases}
$$

We say that $T \in \mathcal{T}_{k}$ is of type $\rho(T)$ and that $T$ is covered if $\rho(T)$ is the all ones vector. $T$ being covered means that $T$ and all of the triangles in $\mathcal{T}_{k}$ that intersect $T$ are contained in $F^{k}\left(T_{0}\right)$. To avoid even further notation, we will use $N(T)$ to denote both the collection of triangles as defined above as well as the set which is the union of those triangles (the carrier of $N(T))$.

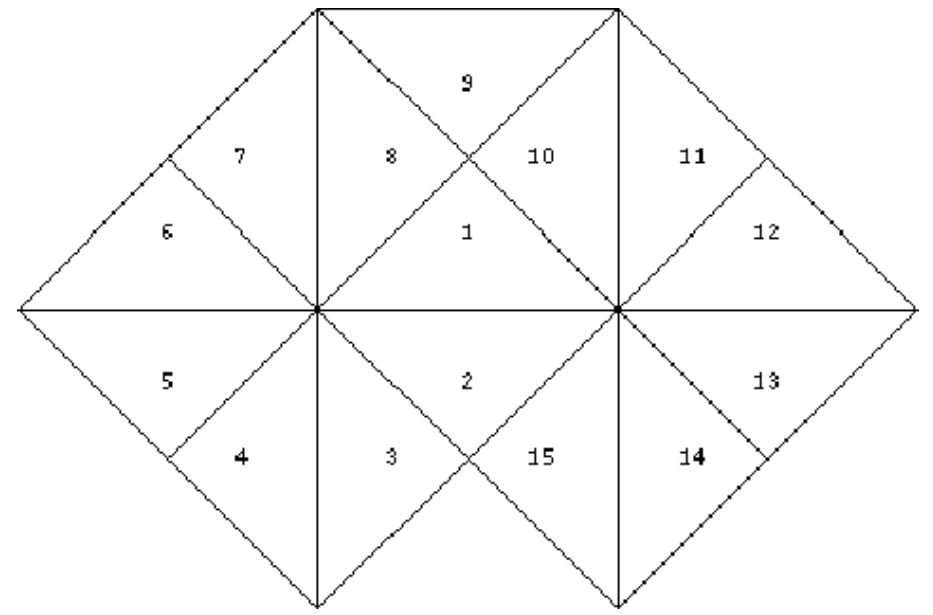

Figure 3. The Neighborhood Ordering

One of the difficulties in dealing with $K$ is that it is not easy to locate points in the interior of $K$ by looking at $F^{k}\left(T_{0}\right)$. One needs to find objects of positive area which persist from iteration to the next, but as soon as a triangle $T$ appears at one level, its interior is discarded at the next level and is replaced by two triangles exterior to $T$. It is not until the iterations have become sufficiently intertwined to create covered triangles that the discarded mass is filled back in by adjacent triangles. Through computer experimentation we discovered that covered triangles do not exist until the 14th iteration, and only 8 of the $2^{14}$ triangles that comprise $F^{14}\left(T_{0}\right)$ are covered! However, if a triangle $T \in \mathcal{T}_{k}$ is covered, then $T$ is the union of two triangles in $\mathcal{T}_{k+1}$ which are also covered. It follows that int $T \subset F^{k+m}\left(T_{0}\right)$ for all $m>0$, so that int $T \subset$ int $K$. We record this and a few other elementary observations about neighborhoods in the next Proposition. 


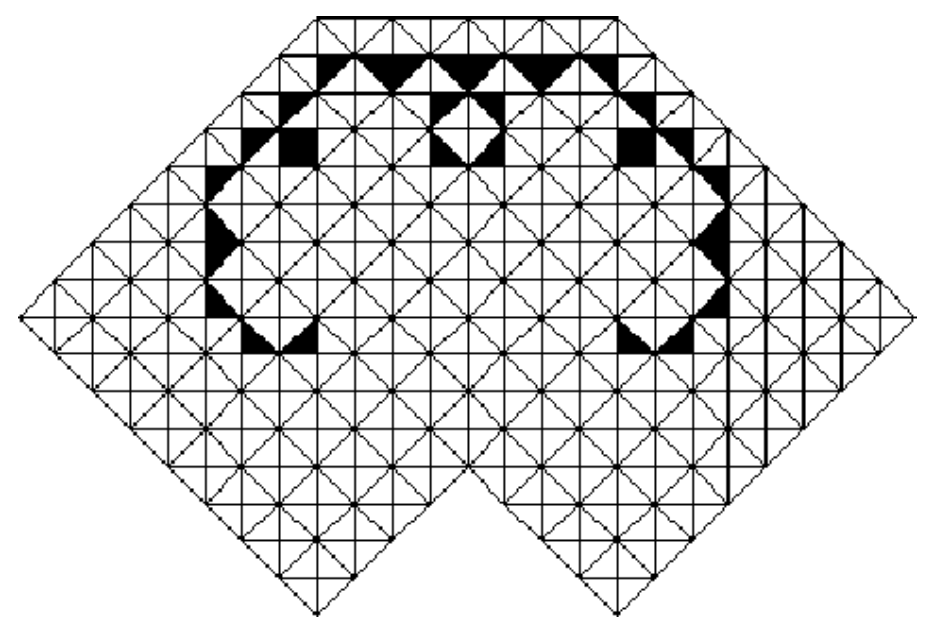

Figure 4. $N_{5}$ And $F^{5} T_{0}$

\subsection{Proposition.}

(1) Let $N_{0}=N\left(T_{0}\right)$. Then $F\left(N_{0}\right) \subset N_{0}$, and therefore $K \subset N_{0}$.

(2) If $T \in \mathcal{T}_{k}$ and $T^{\prime} \subset T$ for some $T^{\prime} \in \mathcal{T}_{k+1}$, then $N\left(T^{\prime}\right) \subset N(T)$.

(3) If $T \in \mathcal{T}_{k}$ is covered and $T^{\prime} \subset T$ for some $T^{\prime} \in \mathcal{T}_{k+1}$, then $T^{\prime}$ is covered.

(4) If $T \in \mathcal{T}_{k}$ is covered, then int $T \subset$ int $K$.

(5) If $v$ is a vertex of any triangle $f_{I_{k}}\left(T_{0}\right) \subset F^{k}\left(T_{0}\right)$, it is also a vertex of some triangle $f_{I_{k+1}}\left(T_{0}\right) \subset F^{k+1}\left(T_{0}\right)$, and therefore $v \in K$.

As we mentioned above, Lévy [L1] observed that the Dragon $K$ tiles the plane, and the tiles occur in the following way. For each triangle $T_{a} \in \mathcal{T}_{0}$ there is an $I F S F_{a}$ whose similarities are related to $T_{a}$ in the same way that $f_{1}$ and $f_{2}$ are related to $T_{0}$. At each stage, the $F_{a}^{k}\left(T_{a}\right)$ tile the plane, and the limits $\left\{K_{a}\right\}$ give a tiling by copies of the Dragon.

Now recall our definitions $B_{k}=\left\{I_{k} \in \Omega_{k} \mid f_{I_{k}}\left(T_{0}\right) \cap \partial F^{k}\left(T_{0}\right) \neq \emptyset\right\}$ and $A_{k}=\left\{I_{k} \in\right.$ $\left.\Omega_{k} \mid f_{I_{k}}(K) \cap \partial K \neq \emptyset\right\}$.

\subsection{Proposition. $B_{k} \subset A_{k}$.}

Proof. Note that $B_{k}$ is the set of $I_{k}$ such that $f_{I_{k}}\left(T_{0}\right)$ is not covered. Suppose that $I_{k} \in B_{k}$, and let $T=f_{I_{k}}\left(T_{0}\right)$. Then for some $T^{\prime} \in N(T), T^{\prime} \nsubseteq F^{k}\left(T_{0}\right)$ and thus $T^{\prime} \subset F_{a}^{k}\left(T_{a}\right)$ for some other tile at the $k$ th level. Let $v$ be a vertex common to $T$ and $T^{\prime}$. By 4.3 (5) applied to both $F$ and $F_{a}, v \in K \cap K_{a}$, so $v \in \partial K$. Since $v=f_{I_{k}}(w)$ for some vertex $w$ of $T_{0}$, $v \in f_{I_{k}}(K)$, so $f_{I_{k}}(K) \cap \partial K \neq \emptyset$, and $I_{k} \in A_{k}$.

It follows that $\left|B_{k}\right| \leq\left|A_{k}\right|$. By using $B_{n}$ in two ways, we can compute $\operatorname{dim}_{H} \partial K$. Let $\beta=\lim _{k \rightarrow \infty}\left|B_{k}\right|^{1 / k}$.

\subsection{Theorem.}

$$
\operatorname{dim}_{H} \partial K=\frac{\ln (\beta)}{\ln (\sqrt{2})} .
$$

Moreover, $0<H^{\alpha}(\partial K)<+\infty$ where $H^{\alpha}(A)$ is the Hausdorff $\alpha$-dimensional measure and $\alpha=\frac{\ln (\beta)}{\ln (\sqrt{2})}$. 
Proof. Since $\beta \leq \alpha$, we get

$$
\frac{\ln (\beta)}{\ln (\sqrt{2})} \leq \operatorname{dim}_{H} \partial K
$$

from the formula at the end of $\S 3$. On the other hand, we can also use $\beta$ to get an upper bound for the box counting dimension of $\partial K$, which is equal to the Hausdorff dimension by Theorem 2.2. Let $N_{k}$ denote the the set of triangles $T$ in the subdivision of $N_{0}$ induced by $\mathcal{T}_{k}$. For such a $T$, it is clear that if $\rho(T)$ is the all zeros vector, then int $T \cap K=\emptyset$ and if $T$ is covered, int $T \cap \partial K=\emptyset$. Thus, $\partial K \subset \bigcup\left\{T \in N_{k} \mid \rho(T)\right.$ is not constant $\}$. By expanding to neighborhoods, we get $\partial K \subset \bigcup\left\{N(T) \mid N(T)\right.$ contains some $\left.f_{I_{k}}\left(T_{0}\right), I_{k} \in B_{k}\right\}$. There are at most $15\left|B_{k}\right|$ such $N(T)$, so for each $k, \partial K$ is covered by at most $15\left|B_{k}\right|$ sets of diameter $\leq 3(\sqrt{2})^{-k}$. Therefore, the box counting dimension of $\partial K$ is less than or equal to

$$
\lim _{k \rightarrow \infty}-\frac{\ln \left(15\left|B_{k}\right|\right)}{\ln \left(3(\sqrt{2})^{-k}\right)}=\frac{\ln (\beta)}{\ln (\sqrt{2})} .
$$

The referee pointed out the second part of the theorem. It is one of the advantages of the approach of Strichartz and Wang [SW] using the graph directed construction of Mauldin and Williams $[\mathrm{MW}]$ that one can claim that $0<H^{\alpha}(\partial K)<+\infty$. It also follows from $[\mathrm{MW}]$ and the fact in this paper that the matrix $M$ used in arriving at the asymptotic estimate of $\left|B_{k}\right|$ is irreducible.

\section{$\S 5$. Computational issues}

In the light of Theorem 4.5, our goal is to understand the asymptotic behavior of the number $\left|B_{k}\right|$ of triangles in $F^{k}\left(T_{0}\right)$ which are not covered. To this end, we need to study the neighborhood structures introduced in $\S 4$. We will index the binary vectors of length 15 by integers between 0 and $2^{15}-1=32767$ by the correspondence $\left(x_{1}, x_{2}, \cdots, x_{15}\right) \Leftrightarrow$ $\sum_{i=1}^{15} x_{i} 2^{i-1}$. Thus we could indicate that $T$ is covered by writing $\rho(T)=32767$. Recall that each $T \in \mathcal{T}_{k}$ is the union of two triangles $T_{1}$ and $T_{2}$ in $\mathcal{T}_{k+1}$, where we let $T_{1}$ be the triangle that contains the left vertex of $T$, and $T_{2}$ contains the right vertex. If we know the neighborhood type for $T$, it is simple (but tedious) to write down the neighborhood types for $T_{1}$ and $T_{2}$. We have

5.1 Proposition. For $T \in \mathcal{T}_{k}$, if

$$
\rho(T)=(x 1, x 2, x 3, x 4, x 5, x 6, x 7, x 8, x 9, x 10, x 11, x 12, x 13, x 14, x 15),
$$

then

$$
\rho\left(T_{1}\right)=(x 8, x 1, x 9, x 8, x 10, x 9, x 1, x 10, x 15, x 3, x 2, x 5, x 4, x 7, x 6),
$$

and

$$
\rho\left(T_{2}\right)=(x 10, x 1, x 12, x 11, x 14, x 13, x 2, x 15, x 3, x 8, x 1, x 9, x 8, x 10, x 9) .
$$

Proposition 5.1 gives a way to compute $\left|B_{k}\right|$ in principle. Let $V(k)$ be the $2^{15}$ long vector such that $V(k)_{i}=$ number of $T \in N_{k}$ such that $\rho(T)=i$. Then let M be the $2^{15} \times 2^{15}$ 
matrix with $M_{i, j}=t$, if a triangle of type $i$ gives rise to $t$ subtriangles with type $j$. For example, $M_{0,0}=2, M_{32767,32767}=2, M_{0, j}=0, i>0$, and $M_{32767, j}=0, j<32767$. $M$ can be constructed from the information in 5.1. Then $M$ has the property that $V(k+1)=V(k) \cdot M$, so if $J$ is the column vector with $J_{i}=1$ if $i$ is odd and less than 32767, 0 otherwise, then $\left|B_{k}\right|=V(0) \cdot M^{k} \cdot J$ and we could study the asymptotic behavior of $\left|B_{k}\right|$ via the eigenvalues of $M$. Fortunately, we do not have to go to such extremes. A little thought shows that not every one of the $2^{15}$ neighborhood types can occur. Our computations determined that, in fact, relatively few types actually do occur.

The only triangle in $F^{0}\left(T_{0}\right)$ is $T_{0}$ itself, so the neighborhood types for the fifteen triangles in $N_{0}$ are encoded by $1,2,2^{2}, \cdots, 2^{14}$. For example, $T_{0}$ is triangle 1 in its neighborhood, so $\rho\left(T_{0}\right)=1$. For a more revealing example, consider the triangle $T$ with vertices $\{(0,0),(0,1),(1 / 2,1 / 2)\}$. It is number 8 in the neighborhood of $T_{0}$, but $T_{0}$ is number 10 in $N(T)$. Therefore, $\rho(T)=2^{(10-1)}=512$. If we let $S_{k}=\left\{\rho(T) \mid T \in N_{k}\right\}$, then, $S_{0}=\left\{2^{k}, 0 \leq k \leq 14\right\}$. By applying the formulas above for $\rho\left(T_{1}\right)$ and $\rho\left(T_{2}\right)$, we can compute $S_{1}, S_{2}$, and so on. Again, we determined by computation that $S_{19}=S_{20}$, so that $S_{k+19}=S_{19}$ for all $k>0$. Let $S_{\infty}$ be this common "stable" set of neighborhood types, $S_{\infty}=\left\{s_{1}, s_{2}, \cdots, s_{752}\right\}$. (We will discuss the order on $S_{\infty}$ below.) The cardinality of $S_{\infty}$ is 752 , so we can perform the analysis indicated above with state vector and transition matrix of manageable size. For $k \geq 19$, let $V(k)$ be the vector of length 752 whose $i$ th entry is the number of triangles in $N_{k}$ which have type $s_{i}$. Let $M$ be the $752 \times 752$ matrix with $M_{i, j}=t$, if a triangle of type $s_{i}$ gives rise to $t$ subtriangles with type $s_{j}$. Then as above, $V(k+1)=V(k) \cdot M$, and if $J$ is the column vector whose $i$ th entry is 1 if $s_{i}$ is coded by an odd number $\neq 32767$, and 0 otherwise, then

$$
\left|B_{k}\right|=V(k) \cdot M \cdot J
$$

so that

$$
\left|B_{19+k}\right|=V(19) \cdot M^{k} \cdot J .
$$

The types in $S_{\infty}$ have a nice structure. The types 0 and 32767 are absorbing in that each gives rise to two triangles of the same type at the next level. Let $S_{a}=\{0,32767\}$; let

$$
S_{t}=\{4,8,9,16,32,64,66,128,512,1024,1026,2048,4096,8192,8193,16384\} ;
$$

and let $S_{e}=S_{\infty} \backslash\left(S_{a} \cup S_{t}\right)$. It can be shown by computation that if we order the types in $S_{\infty}$ so that $s_{i}<s_{j}<s_{k}$ if $s_{i} \in S_{t}, s_{j} \in S_{e}, s_{k} \in S_{a}$, then $M$ has the block form

$$
M=\left(\begin{array}{ccc}
P & Q & R \\
0 & C & L \\
0 & 0 & I
\end{array}\right),
$$

where $C$ is a $734 \times 734$ matrix and $I$ is the $2 \times 2$ identity matrix. Write $V(k)=\left(V_{t}(k), V_{e}(k), V_{a}(k)\right)$, and let

$$
J=\left(\begin{array}{c}
J_{t} \\
J_{e} \\
J_{a}
\end{array}\right)
$$


where the blocking is consistent with that of $M$. Then we have

$$
\left|B_{19+k}\right|=\left(V_{t}(k), V_{e}(k)\right) \cdot\left(\begin{array}{cc}
P & Q \\
0 & C
\end{array}\right)^{k} \cdot\left(\begin{array}{c}
J_{t} \\
J_{e}
\end{array}\right)
$$

The matrix $P$ can be seen to be a permutation matrix, and $C$ is primitive in the sense of Perron-Frobenius theory [BP], i.e., all entries of some power of $C$ are positive. In fact, a calculation shows that $C^{25} \gg 0$. It follows [BP, Theorem 2.4.1] that $C$ has an eigenvalue $\lambda$

equal to its spectral radius, and that $\lim _{k \rightarrow \infty} \frac{1}{\lambda^{k}} C^{k}=D$, where $D$ is a positive matrix. In our case, $\lambda>1$, so

$$
\lim _{k \rightarrow \infty} \frac{1}{\lambda^{k}}\left(\begin{array}{cc}
P & Q \\
0 & C
\end{array}\right)^{k}=\lim _{k \rightarrow \infty}\left(\begin{array}{cc}
\frac{1}{\lambda^{k}} P^{k} & \frac{1}{\lambda^{k}} P^{k-1} Q \\
0 & \frac{1}{\lambda^{k}} C^{k}
\end{array}\right)=\left(\begin{array}{cc}
0 & 0 \\
0 & D
\end{array}\right)
$$

Therefore,

$$
\lim _{k \rightarrow \infty} \frac{\left|B_{k+19}\right|}{\lambda^{k+19}}=\frac{1}{\lambda^{19}} V_{e}(19) \cdot D \cdot J_{e}=q>0
$$

Then we have

$$
\beta=\lim _{m \rightarrow \infty}\left|B_{m}\right|^{1 / m}=\lim _{m \rightarrow \infty} \lambda q^{1 / m}=\lambda
$$

By $\S 4$,

$$
\operatorname{dim}_{H} \partial K=\frac{\ln (\lambda)}{\ln (\sqrt{2})} .
$$

Floating point computations using the power method give the estimate $\lambda \approx 1.954776399$. That approximation for $\lambda$ gives $\operatorname{dim}_{H} \partial K \approx 1.934007183$. According to [BP, Theorem 2.2.35], the spectral radius $r$ of an irreducible matrix $L$ satisfies $u \leq r \leq U$, where $u$ and $U$ are the minimum and maximum row sums of $L$, so it follows that our $\lambda$ satisfies $u_{k}^{1 / k} \leq \lambda \leq U_{k}^{1 / k}$ for each $k$, where $u_{k}$ and $U_{k}$ are the minimum and maximum row sums of $C^{k}$. We computed $u_{30}=67936360$ and $U_{30}=727212953$ using integer arithmetic, which gives the rigorous estimate $1.824190<\lambda<1.974189$. Thus $\lambda$ is provably greater than $\sqrt{2}$, and the Hausdorff dimension of $\partial K$ is greater than 1 . Thus, the boundary of the Dragon has Hausdorff dimension greater than 1, and Edgar's question is answered in the affirmative.

\section{REFERENCES}

[B] C. Bandt, Self-similar sets 5. Integer matrices and tilings of $\mathbf{R}^{n}$, Proc. Amer. Math. Soc. 112 (1991), 549-562.

[BP] A. Berman and R. Plemmons, Nonnegative Matrices in the Mathematical Sciences, Academic Press, 1979.

[DKV] P. Duvall, J. Keesling, and A. Vince, The Hausdorff dimension of the boundary of a self-similar tile, Jour. London Math. Soc. (to appear).

[E1] G. A. Edgar, Measure, Topology, and Fractal Geometry, Springer-Verlag, 1990.

[E2] G. A. Edgar, Classics on Fractals, Addison-Wesley.

[F1] K. J. Falconer, Sub-self-similar sets, Trans. Amer. Math. Soc. 347 (1995), no. 8, 3121-3129. 
[F2] K. J. Falconer, Fractal Geometry Mathematical Foundations and Applications, John Wiley and Sons, 1990.

$[\mathrm{H}] \quad$ J. E. Hutchinson, Fractals and self similarity, Indiana Univ. Math. J. 30 (1981), 713-747.

[K] J. Keesling, The boundary of a self-similar tile in $\mathbf{R}^{n}$, Top. Appl. 94 (1999), 195-205.

[L1] P. Lévy, Les courbes planes ou gauches et les surfaces composée de parties semblales au tout, Journal de l'École Polytechnique (1938), 227-247,249-291.

[L2] P. Lévy, Plane or space curves and surfaces consisting of parts similar to the whole, Classics on Fractals (G. Edgar, ed.), Addison-Wesley, pp. 181-239.

[MW] D. Mauldin and S. Williams, Hausdorff dimension in graph directed constructions, Trans. Amer. Math. Soc. 309 (1988), 811-829.

[SW] R. Strichartz and Y. Wang, Geometry of self-affine tiles I, (preprint).

Department of Mathematical Sciences, University of North Carolina at Greensboro, Greensboro, NC 27412, USA

E-mail address: duvallp@uncg.edu

University of Florida, Department of Mathematics, P.O. Box 118105, 358 Little Hall, Gainesville, FL 32611-8105, USA

E-mail address: jek@math.ufl.edu 